\title{
Effect of Guhong injection combined with atorvastatin on angina pectoris of coronary heart disease and its influence on Hemorheology and oxidative stress
}

\author{
Ze Wang ${ }^{1}$, Li Du*2, Mengyuan $\mathrm{Hu}^{1}$ \\ ${ }^{1}$ Baogang Hospital, Baotou, Inner Mongolia, China \\ 2 Jiuyuan Qu Hospital, Baotou, Inner Mongolia, China
}

Received: October 30, 2020

DOI: $10.5430 /$ dcc.v7n3p12
Accepted: December 22, 2020 Online Published: January 3, 2021

URL: https://doi.org/10.5430/dcc.v7n3p12

\begin{abstract}
Objective: To explore the effect of Guhong injection combined with atorvastatin on CHD patients with angina pectoris and its influence on hemorheology and oxidative stress.

Methods: 80 CHD patients with angina pectoris admitted to our hospital from December 2018 to December 2019 were randomly divided into the observation group ( 40 cases) and the control group ( 40 cases) by use of the random number table. The patients in the control group were treated with atorvastatin, while those in the observation group were treated with Guhong injection on the basis of the control group. The course of treatment was 2 weeks for each group. The treatment effect, the frequency of angina pectoris seizure and its duration before and after treatment, the changes of hemorheology indexes and oxidative stress and the occurrence of adverse reactions were compared between the two groups.

Results: the total effective rate of the observation group (92.50\%) was higher than that of the control group (70.00\%) $(p<$ .05). The frequency of angina pectoris seizure and its duration in these two groups after treatment were lower than those before treatment (Observation group: $t=25.588,23.009$; Control group: $t=16.587,16.263 ; p<.05$ ); After treatment, the frequency of angina pectoris seizure and its duration in the observation group were lower than those in the control group $(t=15.191,9.425 ; p$ $<.05)$. In the observation group, the hemorheology indexes after treatment were lower than those before treatment $(t=6.742$, $15.224,6.983,23.537, p<.05)$; the hemorheology indexes after treatment in the observation group were lower than those in the control group $(t=5.201,13.913,5.539,13.745 ; p<.05)$. In the observation group, the level of serum SOD after treatment was higher than that before treatment, while the levels of LPO and MDA after treatment were lower than those before treatment $(t=$ $10.839,6.924,8.466 ; p<.05)$; the level of serum SOD after treatment in the observation group was higher than that in the control group, while the levels of LPO and MDA after treatment in the observation group were lower than those in the control group $(t=$ $6.171,6.432,5.394 ; p<.05)$. The incidence of adverse reactions in the observation group was lower than that in the control group $(p<.05)$.
\end{abstract}

Conclusions: Guhong injection combined with atorvastatin has an obvious clinical effect on CHD patients with angina pectoris, and it can improve hemorheology indexes and oxidative stress reaction with fewer adverse reactions.

Key Words: Guhong injection, Atorvastatin, CHD with angina pectoris, Efficacy, Hemorheology, Oxidative stress

*Correspondence: Li Du; Email: nmgbgyyyy@ 163.com; Address: Jiuyuan Qu Hospital, Baotou, Inner Mongolia 014040, China. 


\section{INTRODUCTION}

Coronary heart disease is one of commonly seen cardiovascular diseases, and CHD with angina pectoris is a clinical syndrome manifested by myocardial ischemia and acute or transient ischemia-hypoxia in myocardial cells caused by hemadostenosis on the basis of coronary atherosclerosis. ${ }^{[1,2]}$ According to the statistics, the incidence of CHD with angina pectoris is increasingly upregulated. The clinical manifestations are mainly chest pain, radiating pain and chest distress, sometimes accompanied by tachypnea, palpitation and shortness of breath etc., seriously affecting patients' physical and mental health and life quality. ${ }^{[3,4]}$ Currently, the western medicine treatment for CHD patients with angina pectorisis is mainly focused on the conventional symptomatic treatment. Even though it is able to relieve clinical symptoms, it cannot fundamentally cure the disease. ${ }^{[5]}$ Therefore, it is important to find a safe and effective method for the treatment of CHD with angina pectorisis. ${ }^{[6]}$ This study was designed to explore the effect of Guhong injection combined with atorvastatin on CHD patients with angina pectoris and its influence on hemorheology and oxidative stress.

\section{DATA AND METHODS}

\subsection{General information}

According to the relevant diagnostic standards in Denomination and Diagnostic Criteria for Ischemic Heart Disease, ${ }^{[7]} 80$ CHD patients with angina pectoris admitted to our hospital from December 2018 to December 2019 were selected and randomly divided into the observation group (40 cases) and the control group (40 cases) by use of the random number table. The observation group was made up of 28 cases of male patients and 12 cases of female patients, aged 63-73 $(69.39 \pm 6.57)$, with a course of $2-13(6.37 \pm 1.78)$ years. The control group contained 27 cases of male patients and 13 cases of female patients, aged 61-74 (68.18 \pm 5.98$)$, with a course of $2-15(7.08 \pm 1.65)$ years. There was no statistically significant difference in general data between two groups $(p$ $>.05)$.

\subsection{Inclusion and exclusion criteria}

Inclusion criteria: 1) patients who conformed to the diagnostic standards of CHD with angina pectoris; 2) patients who were no less than 40 years old and no more than 75 years old; 3) patients who signed informed consent forms.

Exclusion criteria: 1) chest pain resulted from other types of heart diseases and causes; 2) patients who took drugs affecting the efficacy within 2 weeks before the study; 3 ) patients with severe pulmonary, renal or hepatic insufficiency; 4) patients with mental diseases; 5) patients with allergic constitution.

Published by Sciedu Press

\section{METHODS}

Both groups of patients were given the conventional treatment of the western medicine, including oxygen uptake, bed rest, NO donors drugs, $\beta$-receptor blockers, LMWH, statins and aspirin enteric-coated tablets etc.. On that basis, the control group of patients were required to orally take atorvastatin (Manufacturer: Guangdong Baike Pharmaceutical Co., Ltd.; Specification: 10 mg; SFDA Approval No.: H20120021; Batch No.: 1607282), the dose was $20 \mathrm{mg}$ per time a day. On the basis of the control group, each patient in the observation group was additionally given $20 \mathrm{ml}$ of Guhong injection dissolved in $250 \mathrm{ml}$ of normal saline by ivgtt, one time per day. The course of treatment was 2 weeks for each group.

\subsection{Criteria of the therapeutical effect}

1) Cured: the cardiac function was improved to Grade 2 , with the main manifestations almost disappeared without the use of nitroglycerin; 2) effective: the cardiac function was improved to Grade 1, with the main manifestations improved, with the dose of nitroglycerin reduced by no less than 50\%; 3 ) ineffective: the cardiac function was not improved, and the main manifestations showed no improvements, with the dose of nitroglycerin reduced by no more than $50 \%$, or even increased.

\subsection{Indicator observation}

It was required to 1) observe the frequency of angina pectoris seizure and its duration in these two groups before and after treatment; 2) observe the changes of hemorheology indexes in these two groups before and after treatment, including high blood viscosity (HBV), low blood viscosity (LBV) and plasma viscosity (PV) and fibrinogen (FIB): $4 \mathrm{ml}$ of elbow venous blood was taken from each patient to isolate plasma and whole blood samples for the measurement use with the help of blood rheometer; 3 ) observe the changes of oxidative stress, such as superoxide dismutase (SOD), lipid peroxide (LPO) and malondialdehyde (MDA): $3 \mathrm{ml}$ of elbow venous blood was taken from each patient before and after treatment respectively to centrifuge and isolate the serum (Centrifugal radius: $10 \mathrm{~cm}$, centrifugation time: $10 \mathrm{~min}$ ), and then store the sample at $-20^{\circ} \mathrm{C}$ for the measurement use; 4) observe the adverse reactions in the two groups.

\subsection{Statistical treatment}

The data acquired from this study were imported into SPSS22.0 software for the statistical use. The measurement data were represented by mean \pm standard deviation. All the data in this study fitted to normal distribution, with the use of $t$-test. The categorical data were compared by use of chi-square test. The difference $(p<.05)$ was of statistical significance. 


\section{Results}

\subsection{The comparison in the therapeutic effect between} two groups

The total effective rate of the observation group $(92.50 \%)$ was higher than that of the control group $(70.00 \%)(p<.05)$, see Table 1 for details.

\subsection{The comparison in the frequency of angina pectoris seizure and its duration between two groups}

There were no statistically significant differences in the frequency of angina pectoris seizure and its duration before treatment between two groups $(t=0.421,0.431, p>.05)$; the frequency of angina pectoris seizure and its duration in these two groups before treatment were lower than those after treatment (Observation group: $t=25.588,23.009$; Control group: $t=16.587,16.263 ; p<.05)$; the frequency of angina pectoris seizure and its duration after treatment in the observation group were lower than those in the control group $(t=15.191,9.425 ; p<.05)$, see Table 2 for details.

\subsection{The comparison in the changes of hemorheology in-} dexes between two groups

There was no statistically significant difference in the changes of hemorheology indexes before treatment between two groups $(t=0.132,0.615,0.302,0.409 ; p>.05)$; there were no obvious changes in hemorheology indexes before and after treatment in the control group $(t=0.763,1.512$, $0.748,0.964 ; p>.05)$; the hemorheology indexes in the observation group after treatment were lower than those before treatment $(t=6.742,15.224,6.983$ and 23.537; $p<.05)$; the hemorheology indexes after treatment in the observation group were lower than those in the control group $(t=5.201$, $13.913,5.539,13.745 ; p<.05)$, see Table 3 for details.

Table 1. The comparison in the therapeutic effect between two groups

\begin{tabular}{llllll}
\hline Group & $\mathbf{n}$ & Cured (\%) & Effective (\%) & Ineffective (\%) & Total Effective Rate (\%) \\
\hline Observation Group & 40 & $28(70.00)$ & $9(22.50)$ & $3(7.50)$ & $37(92.50)$ \\
Control Group & 40 & $20(50.00)$ & $8(20.00)$ & $12(30.00)$ & $28(70.00)$ \\
$\chi^{2}$ Value & - & - & - & - & 6.646 \\
$p$ Value & - & - & - & - & $<.05$ \\
\hline
\end{tabular}

Table 2. The comparison in the frequency of angina pectoris seizure and its duration between two groups $(\bar{X} \pm \mathrm{s})$

\begin{tabular}{|c|c|c|c|c|}
\hline Group & Observation Time & $\mathbf{n}$ & $\begin{array}{l}\text { Frequency of Angina Pectoris } \\
\text { Seizure (time(s)/week) }\end{array}$ & Duration of Seizure (min/time) \\
\hline \multirow{2}{*}{ Observation Group } & Before Treatment & 40 & $7.34 \pm 0.93$ & $8.12 \pm 1.28$ \\
\hline & After Treatment & 40 & $3.16 \pm 0.45^{* \#}$ & $3.08 \pm 0.53^{* \#}$ \\
\hline \multirow{2}{*}{ Control Group } & Before Treatment & 40 & $7.26 \pm 0.76$ & $8.24 \pm 1.21$ \\
\hline & After Treatment & 40 & $4.83 \pm 0.53^{*}$ & $4.51 \pm 0.80^{*}$ \\
\hline
\end{tabular}

Note. ${ }^{*}$ In comparison with the indicators before treatment, $p<.05$; ${ }^{*}$ in comparison with the control group, $p<.05$.

Table 3. The comparison in the changes of hemorheology indexes between two groups $(\bar{X} \pm \mathrm{s})$

\begin{tabular}{lllllll}
\hline Group & Observation Time & $\mathbf{n}$ & HBV $(\mathbf{m P a} \cdot \mathbf{s})$ & LBV $(\mathbf{m P a} \mathbf{s})$ & PV $(\mathbf{m P a} \cdot \mathbf{s})$ & FIB $(\mathbf{g} / \mathbf{L})$ \\
\hline \multirow{2}{*}{ Observation Group } & Before Treatment & 40 & $5.73 \pm 1.20$ & $18.32 \pm 2.19$ & $2.01 \pm 0.53$ & $3.82 \pm 0.56$ \\
& After Treatment & 40 & $4.1 \pm 0.87^{* \#}$ & $11.45 \pm 1.83^{* \#}$ & $1.29 \pm 0.38^{* \#}$ & $1.49 \pm 0.28^{* \#}$ \\
Control Group & Before Treatment & 40 & $5.69 \pm 1.49$ & $18.65 \pm 2.59$ & $1.97 \pm 0.65$ & $3.76 \pm 0.74$ \\
& After Treatment & 40 & $5.45 \pm 1.32$ & $17.83 \pm 2.25$ & $1.89 \pm 0.57$ & $3.58 \pm 0.92$ \\
\hline
\end{tabular}

Note. ${ }^{*}$ In comparison with the indicators before treatment, $p<.05$; ${ }^{\#}$ in comparison with the control group, $p<.05$.

\subsection{The comparison in the changes of oxidative stress between two groups}

There were no statistically significant differences in the changes of SOD, LPO and MDA levels before treatment between two groups $(t=0.777,0.297,0.278 ; p>.05)$. There were no obvious differences in the changes of SOD, LPO and
MDA levels before and after treatment in the control group $(t=0.742,0.786$ and $0.920 ; p>.05)$. In the observation group, the level of serum SOD after treatment was higher than that before treatment, while the levels of LPO and MDA after treatment were lower than those before treatment $(t=$ $10.839,6.924,8.466 ; p<.05)$; the level of serum SOD after 
treatment in the observation group was higher than that in the control group, while the levels of LPO and MDA after treatment in the observation group were lower than those in the control group $(t=6.171,6.432,5.394 ; p<.05)$, see Table 4 for details.

\subsection{The comparison in the incidence of adverse reac- tions between two groups}

The incidence of adverse reactions in the observation group was lower than that in the control group $(p<.05)$, see Table 5 for details.

Table 4. The comparison in the changes of oxidative stress between two groups $(\bar{X} \pm \mathrm{s})$

\begin{tabular}{llllll}
\hline Group & Observation Time & $\mathbf{n}$ & SOD $(\mathbf{U} / \mathbf{L})$ & LPO $(\boldsymbol{\mu m o l} / \mathbf{L})$ & MDA $(\boldsymbol{\mu m o l} / \mathbf{L})$ \\
\hline \multirow{2}{*}{ Observation Group } & Before Treatment & 40 & $63.42 \pm 7.81$ & $5.47 \pm 1.39$ & $5.93 \pm 1.25$ \\
& After Treatment & 40 & $79.93 \pm 5.64^{* \#}$ & $3.79 \pm 0.65^{* \#}$ & $3.71 \pm 1.09^{* \#}$ \\
\multirow{2}{*}{ Control Group } & Before Treatment & 40 & $61.87 \pm 9.90$ & $5.38 \pm 1.32$ & $5.84 \pm 1.62$ \\
& After Treatment & 40 & $64.01 \pm 15.31$ & $5.16 \pm 1.18^{*}$ & $5.49 \pm 1.78^{*}$ \\
\hline
\end{tabular}

Note. ${ }^{*}$ In comparison with the indicators before treatment, $p<.05$; ${ }^{*}$ in comparison with the control group, $p<.05$.

Table 5. The comparison in the incidence of adverse reactions between two groups

\begin{tabular}{llllll}
\hline Group & $\mathbf{n}$ & Gastrointestinal Reaction & Debility & Dizziness & Incidence (\%) \\
\hline Observation Group & 40 & 1 & 1 & 1 & 7.50 \\
Control Group & 40 & 4 & 3 & 4 & 27.50 \\
$\chi^{2}$ Value & - & - & - & - & 5.541 \\
$p$ Value & - & - & - & - & $<.05$ \\
\hline
\end{tabular}

\section{DISCUSSION}

According to the traditional Chinese medicine, CHD with angina pectoris is considered as true heart pain, heart pain and chest discomfort, which is thought to be associated with spleen, renal and hepatic function. ${ }^{[8,9]}$ TCM theory holds that CHD with angina pectoris is probably a type of asthenia in origin and asthenia in superficiality, accompanied by cold congelation, qi stagnation, blood stasis and phlegm turbidity, which can result in heart vessel blockage stasis. Blood stasis is an important pathogenesis. ${ }^{[10]}$ As a type of traditional Chinese and western medicine compound, Guhong injection is made up of aceglutamide and the extracts of safflower, which has an effect of promoting blood circulation for removing blood stasis. Modern pharmacological research indicates that ${ }^{[11,12]}$ aceglutamide is one of acetyl compounds of glutamine. It can take effect on the cardiac conducting system by enhancing the pacing function of $\mathrm{P}$ cells in sinoatrial nodes; the extracts of safflower can effectively eliminate free radicals, suppress the lipid oxidation of cell membranes, enhance the activity of SOD and inhibit platelet aggregation. This type of extracts can also relieve microcirculation disturbance, dilate blood vessels and improve the hemorheology indexes. Atorvastatin, which is one of lipid-lowering drugs, can block the transformation from 3hydroxyl-3-methylglutaryl-coenzyme A to methyldihydroxyvaleric acid, enhance the effect of liver on eliminating low density lipoproteins and then decrease the level of blood

Published by Sciedu Press lipid. This study shows that the total effective rate of the observation group is higher than that of the control group. It is indicated that Guhong injection combined with atorvastatin can acquire a good efficacy. The frequency of $\mathrm{f}$ angina pectoris seizure and its duration after treatment in the observation group are lower than those in the control group, which suggests that Guhong injection combined with atorvastatin can reduce the frequency of angina pectoris seizure and its duration.

Modern medicine holds that oxidative stress and its mediated lipid metabolism and peroxidation reaction play an important role in the occurrence and development of CHD with angina pectoris. ${ }^{[13]} \mathrm{SOD}$, one of anti-oxidative substances, can eliminate free radicals and prevent the tissues from being damaged by peroxidation and free radicals. The changes in SOD content can reflect the anti-oxidative ability. ${ }^{[14]} \mathrm{My}-$ ocardial ischemia in the course of CHD can generate a large number of free radicals, which can bind to the side chains of unsaturated fatty acids and then obviously increase the LPO level in myocardial ischemia. ${ }^{[15]}$ As a terminal product of lipid peroxidation, MDA can reflect the degree of lipid peroxidation and represent the rate and the intensity of lipid peroxidation. ${ }^{[16]}$ This study shows that SOD level after treatment in the observation group is higher than that in the control group, and the levels of LPO and MDA after treatment in the observation group are lower than those in the control group. It is indicated that Guhong injection combined 
with atorvastatin can improve the oxidative stress.

In CHD patients with angina pectoris, the blood keeps in a hypercoagulable state, with an abnormal blood coagulation and fibrinolysin system. ${ }^{[17]}$ In addition, the occurrence of thrombosis and the up-regulated lipid level in CHD patients can lead to the increase of blood viscosity and the decrease of erythrocyte deformability and result in the changes of hemorheology indexes. ${ }^{[18,19]}$ The changes of hemorheology indexes are considered to be the important indicators for evaluating hypercoagulable state, which contribute to the evaluation of patients' condition. ${ }^{[20]}$ This study shows that the levels of hemorheology indexes after treatment in the observation group are lower than those in the control group. It is indicated that Guhong injection combined with atorvastatin can improve the abnormal hemorheology indexes.

In conclusion, Guhong injection combined with atorvastatin has an obvious clinical effect on CHD patients with angina pectoris, and it can improve hemorheology indexes and oxidative stress reaction with fewer adverse reactions.

\section{CONFLICTS OF INTEREST DisClOSURE}

The authors declare they have no conflicts of interest.

\section{REFERENCES}

[1] Hagström E, Norlund F, Stebbins A, et al. Psychosocial stress and major cardiovascular events in patients with stable coronary heart disease. J Int Med. 2018; 283(1): 83-92. PMid:28960596. https://doi.org/10.1111/joim.12692

[2] Mohamad N, Ikhsan ND, Ismail RAR, et al. The Effect of Health Education Program on Knowledge of Coronary Heart Disease (CHD) Among Public in Puncak Alam, Selangor. Advanced Science Letters. 2018; 24(1): 556-558. https://doi .org/10.1166/asl . 2018.1 2069

[3] Sun H, Li XN, Zhang AH, et al. Exploring potential biomarkers of coronary heart disease treated by Jing Zhi Guan Xin Pian using high-throughput metabolomics. RSC Advances. 2019; 9(20): 1142011432. https://doi.org/10.1039/C8RA10557 J

[4] Wang J, Kong W, Bai Y, et al. Analysis on the pathogenesis of CHD with T2DM and simple CHD and risk factors. Chinese Journal of Integrative Medicine on Cardio/Cerebrovascular Disease. 2017; 15(23): 2999-3002.

[5] Bi Y, Wang X, Mao J, et al. Diagnostic recommendations on TCM syndrome of CHD with angina pectoris based on clinical epidemiological investigation. Journal of Traditional Chinese Medicine. 2018; 59(22): 92-95.

[6] Zhang Q, Zhang K, Gu H. Clinical Efficacy of Shexiang Baoxin Pill Combined with Rosuvastatin in Treatment of Angina Pectoris and Influence on Hemorheology and Blood Lipid. Chinese Archives of Traditional Chinese Medicine. 2018; 12(7): 1777-1779.

[7] ISFC. Denomination and Diagnostic Criteria for Ischemic Heart Disease. Chinese Journal of Cardiology. 1981; 9(1): 75-76.

[8] Leng D, Gao E, Li F, et al. Research Progress of Treatment for Angina Pectoris of Coronary Heart Disease. Information on Traditional Chinese Medicine. 2017; 34(4): 119-123.

[9] Liu A. Effect of Xiaoke Tongguan Decoction on the treatment of diabetes mellitus complicated with coronary heart disease and its influence on the levels of high mobility group protein B1 and serum omentin 1. Modern Journal of Integrated Traditional Chinese and Western Medicine. 2018; 27(21): 2307-2310.

[10] Hu L, Yu G, Du Y, et al. Research progress on TCM treatment of CHD with angina pectoris. Hunan Journal of Traditional Chinese Medicine. 2018; 34(5): 183-185.

[11] Yang Y, Ran X, Ran J, et al. Observation on the effect of Guhong injection on the treatment of acute branch atheromatous disease. Mod- ern Journal of Integrated Traditional Chinese and Western Medicine. 2019; 28(20): 2216-2218.

[12] Fu Q, Qi J. Therapeutic Effect of Gu Hong Injection on Angina Pectoris after PCI in Patients with Coronary Heart Disease and its Influence on Inflammatory Reaction and Vascular Endothelial Function. Journal of Clinical Research. 2018; 35(1): 79-82.

[13] Wang S, Zheng X, Sun W. Effects of Ezetimibe and Atorvastatin on Oxidative Stress and Blood Lipid Levels of Patients with Coronary Heart Disease. Progress in Modern Biomedicine. 2017; 17(13): 2470-2473.

[14] Sun H, Shen Q, Zhang K, et al. Association of Serum Level of RBP4 with Oxidative Stress and Carotid Atherosclerosis in Patients with Coronary Artery Disease in the Elderly. Chinese Journal of Cardiovascular Review. 2019; 17(7): 607-610.

[15] Zhang W. Research on the effect of different doses of atorvastatin on CHD with stable angina pectoris, blood-lipid related indicators and oxidative stress in the lipid-lowering therapy. Shaanxi Medical Journal. 2018; 18(3): 395-397.

[16] Zhang Z, Xie Z. Effects of Shensong Yangxin Capsule and Trimetazidine on the Oxidative Stress in Patients with Angina Pectoris. Chinese Journal of Integrative Medicine on Cardio-/Cerebrovascuiar Disease. 2017; 15(19): 2420-2422.

[17] Song Y, Du W, Ma L, et al. Comparative Analysis of the Effects of Ginkgo Flavone Glycosides and Compound Danshen Tablets on Electrocardiogram, Cardiac Function and Hemorheology in Patients with Coronary Heart Disease Angina. World Chinese Medicine. 2017; 12(8): 1764-1766

[18] Qiu D, Wang F, Zhao T. Treatment effect of salvianolateand its influence on the hemorheology and oxidative stress in angina pectoris patients. Anhui Medical and Pharmaceutical Journal. 2018; 18(1): 147-151.

[19] Hu G, Cheng X, Qu B. Effect of modified Yiqi Huoxue decoction combined with the conventional treatment onthe changes of hemorheology indexes in CHD patients with angina pectoris caused by deficiency of qi and blood stasis. Information on Traditional Chinese Medicine. 2017; 34(4): 86-88.

[20] Liu F, Xie H, Zhao M, et al. Clinical Observation on Heat Sensitive Moxibustion Combined with Conventional Drugs in the Treatment of Qi Deficiency and Blood Stasis Type of Angina Pectoris and Its Influence on Blood Rheology and Blood Lipid Level. Journal of Emergency in Traditional Chinese Medicine. 2017; 26(1): 12-14. 